\title{
Preparation and characterization of core@shell nanoparticles of NaGdF4:Er:Pr:Yb@CaF2
}

\author{
Amanda J. Morais* (IC), Emille M. Rodrigues (PG), Italo O. Mazali (PQ), Fernando A. Sigoli (PQ).
}

\begin{abstract}
In order to obtain a sample with an optimized upconversion emission, many core@shell syntheses of NaGdF4:Er:Yb:Pr@CaF2 were done in order to study the influence of different shell thicknesses on the luminescent properties for a possible application in biological systems. Information on the crystalline phase, particle morphologies and the upconversion emission intensity were obtained using: X-Ray Diffraction (XRD), Transmission Electron Microscopy (TEM) and Luminescence Spectroscopy (LS)
\end{abstract}

\section{Key words:}

Lanthanides, luminescence, nanoparticles

\section{Introduction}

Lanthanide ions-doped low-phonon nanoparticles are currently been studied, mainly because their luminescent properties are favorable to obtain useful systems, such as temperature sensors applied in biological areas. (1) One of the phenomena responsible for the development of these systems is the possibility of emission upconversion. This phenomenon comprises the absorption of at least two photons of lower energy by the lanthanide ions followed by emission of higher energy photons. (2) To protect the lanthanide ions from non-radiative decay processes, when the nanoparticles are dispersed in a solvent or in a biological environment, a protective shell layer must be deposit over the optical active core leading to the formation of a hierarchically nanostructured core@shell systems. (1) The shell consists of inorganic compound surrounding the core and may be composed by one or more than one layer with different thicknesses. This project has used a biocompatible shell of $\mathrm{CaF}_{2}$ having a good chemical stability and transparency over the optical window of interest. (3) The project consisted basically of the synthesis and characterization of core@shell nanoparticles $\mathrm{NaGdF}_{4}: \mathrm{Er}: \mathrm{Yb}: \operatorname{Pr} @ \mathrm{CaF}_{2}$ to study the upconversion luminescent properties for a possible use in biological based applications.

\section{Results and Discussion}

To perform the synthesis the trifluoracetates of lanthanides $\left[\mathrm{Ln}\left(\mathrm{OOCCF}_{3}\right)_{3}\right]$, sodium $\left[\mathrm{NaOOCCF}_{3}\right]$ and calcium

$\left[\mathrm{Ca}\left(\mathrm{OOCCF}_{3}\right)_{2}\right]$ were used as precursors. The solvents and stabilizers of the synthesis were oleic acid, oleylamine and 1-octadecene. The first step consisted in verifying which of crystalline phases, cubic or hexagonal, of the core $\mathrm{NaGdF}_{4}$ nanoparticle show the higher upconversion emission intensity. Therefore, the cubic and hexagonal crystalline phases were prepared using different approaches of the the synthetic procedure. According to the phase diagram, an excess of sodium trifluacetate may lead to formation of the hexagonal phase. Powder X-Ray Diffraction (XRD) data show that the phase formation control was possible and only hexagonal or only cubic phase were easily obtained. Electron Transmission Microscopy data indicate that the nanoparticles are spheroidal and that there is an uniform distribution of shape and size. Finally, the upconversion emission spectra show that hexagonal phase containing a thick shell layer has the higest upconversion emission intensity among the prepared nanoparticle systems, suggesting that there is an effective protection of the active lanthanide ions from the nonradiative decay routes.

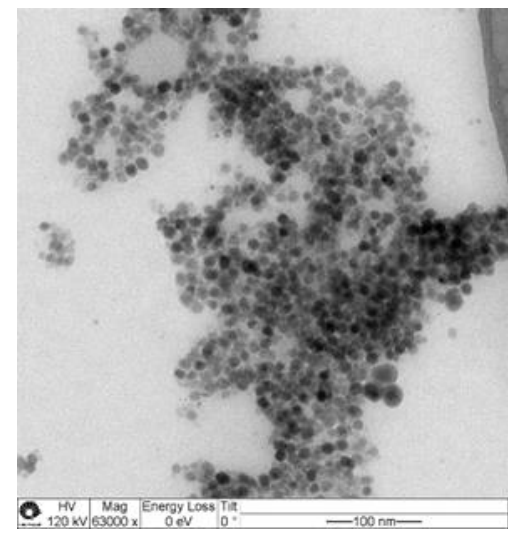

Image 1. Transmission Electron Microscopy image of $\mathrm{NaGdF}_{4}: \mathrm{Er}: \mathrm{Yb}: \operatorname{Pr} @ \mathrm{CaF}_{2}$ nanoparticles.

\section{Conclusions}

The results indicated that, using the proposed methodology, it was possible to obtain the desired crystalline phases of core@shell nanoparticles. The next steps of the research will involve the modification of nanoparticle surface using specific chemical moties in order to disperse these hierarchically nanostructured systems in polar solvents, such as water. The nanoparticle surface chemical functionalization will also allow the anchoring of photosensitizer molecules, such as Rose Bengal, for singlet oxygen photo-generation for possible application on photodynamic therapy (PDT).

\section{Acknowledgement}

CNPq, FAPESP, CAPES, INOMAT and Multi-user Laboratory for Advanced Optical Spectroscopy.

\footnotetext{
1 Gautam, A.; Veggel, F. C. J. M.;Journal of Materials Chemestry, 1 (2013), 5186-5200

2 Johnson, N. J. J.; Veggel, F. C. J. M, Nano Research, 2013, 547, 5186-5200 ${ }^{3}$ Prorok K, Bednarkiewicz a, Cichy B, Gnach a, Misiak M, Sobczyk M, et al. Nanoscale. 2014;6(3):1855-6
} 